\title{
Application of IC Card License for Road Transportation in Commercial Vehicles Supervision and Service
}

\author{
Weiwei Li and Jingyan Gu \\ Research centre of Transport and Logistics, Research Institute of Highway Ministry of Transport, No. 8 Xitucheng Road, Haidian District, \\ Beijing 100088, People's Republic of China
}

\begin{abstract}
IC card electronic license for road transport includes the IC card commercial vehicle's certificate and IC card practitioner's qualification certificate. In China, the IC card electronic license for road transport is the electronic ID card which must be carried by each commercial vehicles and practitioners. This paper briefly introduces the basic situation, data format and security keys architecture of IC card electronic license for road transportation of China. In order to strengthen the supervision and service of commercial vehicles, this paper puts forward the overall application framework of IC card electronic license for road transport. The application examples of IC card license in the supervision of passenger station, dangerous goods transport management, governance overload and logistics park and port area management are discussed. The practical application results show that the application of IC card electronic license for road transport is an important technical means to improve the supervision ability and service quality of the road transportation industry.
\end{abstract}

\section{Introduction}

Commercial vehicles are vehicles engaged in road transport business operations. Road passenger transport management of taxi, tour bus, class line of passenger cars, and road freight transport business trucks, etc. all belong to the commercial vehicles. In road traffic, due to the wide operating range of commercial vehicles, day long operation time, a large number of passenger traffic and a huge amount of logistics operations, commercial vehicles are prone to traffic accidents. Especially the operation of passenger cars, dangerous chemicals transports vehicles and overloaded trucks often cause serious consequences once the accident happened.

In China, the existing commercial vehicles are more than 14 million vehicles, and practitioners are more than 20 million people. With the rapid growth of commercial vehicles and practitioners, the lack of timely and accurate grasp of the operation status of the vehicle, it has been difficult to adapt to the needs of modern road transport management. In addition, the development of China's road transport is still relatively extensive, large-scale, low degree of organization, market concentration is not high. In all the more than seven million and five hundred thousand business households for road freight of the whole country, with five cars below accounted for about $98 \%$, with more than ten cars accounted for less than $1 \%$. Passenger car has five households accounted for about $85 \%$ of the following, with more than ten cars accounted for about $13.5 \%$ [1]. The characteristics of the large scale of China's road transport industry and the dispersion of business operations resulted in a high degree of market operating order specifications, but also which is for the higher management and services requirements to the commercial vehicles.

The information and intellectualization has become the main developing direction of modern traffic safety supervision system and smart card system, global position system (GPS), geographic information system (GIS), computer, wireless communication and Internet technology have been widely integrated application, which is to achieve integrated management of vehicle operation and quality service provides new techniques. Among them smart card system is utilized in many ways in many countries [2]. At present, the Ministry of transport of China is to gradually implement the national IC card electronic license for road transport. IC card electronic license for road transport includes the IC card commercial vehicle's certificate and IC card practitioner's qualification certificate. IC card as the operator carry electronic archives, by virtue of its security to read and write, easy sharing, facilitate the automatic data entry and other advantages, to enhance the level of industry supervision and service provides powerful technical means.

To ensure for realizing IC card for road transport of electronic documents in the provinces and cities a common card in the whole country, the Ministry of transport of China issued relevant standard [3]. The IC card electronic license for road transport must be the key security system, unified appearance style and anticounterfeiting scheme and unified data format unification, 
unified key equipment and technology. At the same time, the Ministry of transport of China unified leadership and co-ordination arrangements for the construction and application of IC card electronic license for road transport.

This organization of this paper is as follows: In section 2, we briefly introduce the technical characteristics of IC card electronic license for road transport in China. After that, in section 3 the general application framework of IC card electronic license for road transport is proposed. In section 4 we discussed the typical application scenario and the application effect is analysed and summarized. Finally, section 5 gives the conclusion with the prospect of further work.

\section{Technical characteristics of IC card electronic license for road transport of China}

\subsection{General technical introduction of IC card electronic license for road transport}

IC card electronic license for road transport in China select non-contact type CPU card. The non-contact CPU card features include: First, the chip and the COS security technology for the CPU card provides the dual security guarantee, with its own operating system CPU card on the computer network system requirements are low, can be achieved offline. IC card electronic license for road transport can be read and write under the network environment, which can be guaranteed under the offline state of a variety of applications. The second is to achieve a real sense of one card and more applications. Each application is independent of each other, and is controlled by the respective key management system. In the IC card electronic license for road transportation set aside the area for other management departments need to read and write. Other management departments can use the original hand-held reading and writing system for IC card electronic license for road transport to read and write, so as to achieve the IC card electronic license for road transport cross sectorial applications. The third is the card storage capacity is large, can provide $1 \mathrm{~K} \sim 64 \mathrm{~K}$ bytes of data storage. The fourth is the card is with an independent security module. Encryption, decryption, and transaction processing are implemented by using the corresponding entity SAM card key, thereby completing the security authentication between the user and the user card.

\subsection{Data format of IC card electronic license for road transport}

The data format of the IC card electronic license for road transport is in accordance with the relevant requirements of the transportation industry standards in China the IC card for road transportation certificate applied unified national style and the unified data format. The information contents of the IC card electronic license for road transport mainly include the basic information file, the basic directory file, the national main application area and the local main application area [3]. Data storage space is 8954 bytes. The basic information file mainly includes the provincial administrative division code identification, vehicle territorial administrative division code, card version number, card initialization date, the card initialization authority and card number and other information. The basic directory file includes the contents of the various types of applications included in the card. The national main application areas include the province of annual audit cases accumulated data file, outside the province inspection application information file information such as the information. The local main application areas include two types of data: one is static data, the other is scalable data. Static data is with the vehicle and personnel basic information about the data, such as vehicle license, road transportation certificate number, license plate number, business qualification, scope, secondary maintenance, technical grade status and owner's name, identity card number and other basic situation and vehicles and the personnel of the picture file. The scalable data is generated in the offline management information, such as fees to pay, annual audit records, records of violation, administrative penalties and other information data.

\subsection{Security keys architecture of IC card electronic license for road transport}

In order to strengthen the security and confidentiality of IC card electronic license for road transportation and key management, IC card electronic license for road transportation key system adopt ministerial and provincial two level management models. Ministerial key includes the ministerial road transport of electronic license business root key and the ministerial road transport of electronic license business master key. Ministerial road transportation electronic certificate business root key can be controlled to produce the ministerial road transport electronic license business master key, card control key, etc. The master key of the ministerial road transport of electronic business license includes road transport of electronic license the read and writes the key, such as IC card for road transportation electronic certificate authentication key, IC card for road transportation electronic license outside the province inspection business application key. Provincial root key is the key of provincial local application. Provincial business key mainly includes local application related key [3]. At present, China's Ministry of transport has established the IC card electronic certificate for road transportation of ministerial key system, to ensure that the IC card electronic license for road transport can achieve to be read and written across the whole country.

\section{General application framework of IC card electronic license for road transport}

In this paper, we offer the overall framework of IC card electronic license for road transport management and application according to the above IC card technology characteristics. IC card electronic license for road transport management and service system is mainly 
composed of ministerial level and provincial level two system. The framework is illuminated in Figure 1.

The ministerial level is responsible for the unified provincial level management of the whole country. The ministerial level key management system is mainly to achieve the management of road transport industry the root key, the generation and management of the national unified reading and writing key, and the secure distribution of provincial key. Relying on the ministerial and provincial road transportation information system network and through the ministerial level road transportation information service system of IC card management function expansion, the Ministry of transport can achieve relevant information about the IC card electronic certificate for road transportation issuing, tracking and summary.

The provincial level is responsible for the issuance and application of IC card electronic license for road transport. Relying on available provincial road transport management system expansion development of IC card issuing and management subsystem, the provincial level key management system can realize the establishment of the IC card system initialization, key management system and IC card application system [3]. IC card initialization system mainly uses the equipment to realize the IC card initialization and initialization key filling, IC card quality inspection and sorting, file structure and the basic information data write functions.

In addition, the ministerial level can rely on the ministry-province networking data exchange platform to build the national IC card electronic license for road transportation black list data exchange and query functions, which can achieve cross regional and transdepartmental linkage inspection, collaborative supervision to provide technical supporting platform.

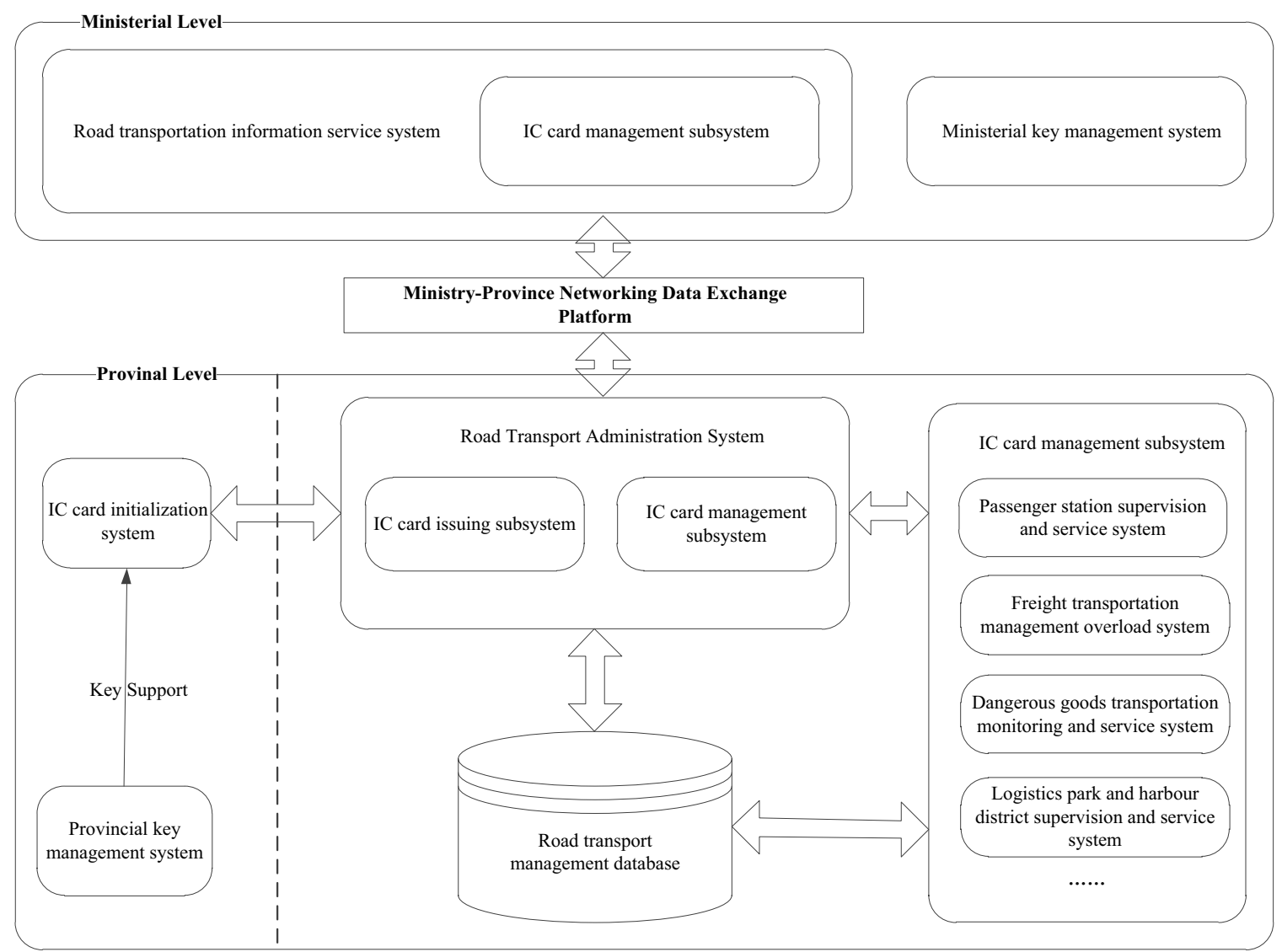

Figure 1. Application framework of IC card electronic license for road transportation.

\section{Typical application scenario analysis of IC card electronic license for road transport}

IC card electronic license for road transportation has been in the passenger vehicle station management, dangerous goods transport management, governance overload, Logistics Park and port area management and other aspects of a wide range of applications. The typical application scenario analysis is as follows.

\subsection{Passenger station supervision and service system}

The IC card electronic license for road transportation as a carrier, by recording bus stop check, security check, inspection certificates, sign up for a class, check the business aspects of management information station, the realization of the entrance and safety supervision station and sign up for a class of whole business process for passenger vehicles. At the same time, IC card as an authoritative carrier of security cases and the entrance 
and exit the station, but also provides strong evidence for road traffic inspection and safety inspection [4].

\subsubsection{Passenger vehicles enter the station verification}

When passenger vehicles enter the antenna reading area, the IC card can be identified by the antenna, and through the background of the vehicle library data to determine the conditions of the vehicle. According to the electronic data inspection results are divided into two categories, in line with the conditions of vehicles to enter the station without stopping. The vehicles which do not meet the conditions will be turned to manual control.

\subsubsection{Car wash, security and class report management}

After allowing the vehicle to enter the station, in the car wash disinfection, routine security checks, classes and other aspects of the use of IC cards to record business information in real time. In addition, the system provides the third party application access interface such as the fingerprint, face recognition, alcohol detection system to effectively monitor the vehicles travel safety. When vehicle class the scheduling system and documents will verify the system data to determine the execution system automatically to sign up for a class of results and push the result to the station LED display. The driving personnel can pass through the LED display clear the contents of the subsequent execution of the task. The document verification system is the automatic information collection and verification of the five kinds of documents, which includes the driver's license, qualification certificate, driving license, and vehicle certificate and line card.

\subsubsection{Passenger vehicle departure verification}

When the passenger vehicle is ready to leave the station, once the vehicle enter the antenna area and the antenna read the IC card, all the information includes the car flight number and number of passengers and other information through the antenna will be written into the IC card. All the implementation results will be displayed in the LED display of the station. At the same time, the system allows the vehicle to leave the station without a stop. Exit of station will write the vehicle trips, and number of passengers and other information into the IC card through the antenna, which will facilitate management by mobile terminals.

\subsection{Freight transportation overload management system}

The focus of freight transportation overload management is to focus on the source and road control. The IC card electronic license for road transport can be used as the sole identity card of the freight transportation vehicle. The road transportation certificate, qualification certificate, driving all the cards, card of drive a vehicle, weighing information such as information entry in the IC card, can realize multi card into single unity as the basis for supervision [5].

\subsubsection{Supervision of the source of cargo transport}

IC card electronic license for road transportation is the permit document for source of freight transport. Cargo transport vehicles must hold IC cards to enter the source enterprise loading. In the source of freight source goods enterprise will be responsible for writing the loading of information in road transportation IC card. All freight source units on government publicity will be all included in the road transportation IC card source overload management system. In the scattered goods transport is relatively concentrated area the goods concentrated weigh point will be set up to implement IC card management to achieve full coverage of the source.

\subsubsection{Roadway checks at toll station or overload inspection station}

The control system of overload and highway toll system for docking can be in the toll station to achieve the regulation of vehicles. When the overload vehicles passed the weighing system, the software can display the information overload and overrun. At the same time, the display LED set on the highway toll plaza will show the ban line information to the driver. When the overloaded vehicles entered into the toll lanes, the toll collection system will be on the toll again prompted to limit information. If the toll collectors insist on forcing the pass card, then the system will automatically upload the vehicle information to the monitoring system.

\subsection{Dangerous goods transportation monitoring and service system}

Combined IC card electronic license for road transportation with the record risk goods transportation electronic waybills, transport of dangerous goods vehicles running state, loading dangerous goods of the type and number, driving route, and the driver, supercargo and information management for process monitoring, the transport of dangerous goods from the vehicle scheduling, loading, transportation and unloading will realize the closed-loop management of whole process, which will reduce the safety hidden danger to the greatest extent [6].

\subsubsection{Controllable source and traceable responsibility}

Before the transportation, the carrier online fill in the waybill information and automatically download to the road transportation IC card through reading and writing device or vehicle terminal. Prior to the shipment of the goods the shipper will verify the transport vehicles and personnel qualification of the transport of dangerous goods qualification enterprises, vehicles and personnel engaged in the transport of dangerous goods by reading 
the IC card electronic license, which will prevent the illegal operation behaviour on the bills to fill in the content confirm. In addition, through the system you can check whether the source of the company commissioned a carrier of the carrier's transport of goods to provide strong evidence of responsibility after the accident occurred.

\subsubsection{Real time monitoring and reliable emergency response}

Combined with the electronic waybill information and the real-time vehicle route and track, management personnel can know the real-time dangerous goods of each type, weight and objective, which will provides effective means in real time for industry statistics analysis and emergency disposal. When unexpected events occur, industry management department query electronic waybills, obtain goods category, the number of information, and combining with GIS intuitive understanding of dangerous goods transport vehicles location of the surrounding topography, lakes, villages and other information, for emergency and accident rescue plan is formulated to provide effective technical support.

\subsection{Logistics Park and harbour district supervision and service system}

\subsubsection{Freight market credit information service}

IC card electronic license for road transportation is an important support in the transport market for the commercial vehicles and practitioners of the identity of the qualification certification. In logistics parks and port freight yards, by authorization authentication terminal, national vehicle and personnel database and IC card electronic license for road transportation by $\mathrm{CA}$ authentication technology, provide transport practitioners member, vehicle certification service for the public through IC card authentication and network authentication mode. The system will be convenient for users to check the authenticity and legitimacy of the relevant documents, and select the normal operation of the vehicle and professional personnel commissioned by the transport business. This service will effectively regulate the transport market, maintaining the normal order of the transport market.

\subsubsection{Park and station management and service}

On national highway transport freight hub station field, logistics park and key port deployed the card reading device, and in the IC card electronic license for road transportation to increase field management and service function, can realize field station one card multi-service system, so as to facilitate the vehicle certification and in and out of the station management. Logistics Park and port will be as a national freight service system of merchants, for the cardholder to provide parking, accommodation, catering, repair, and picking and preferential, consumption points and other value-added services, cardholders with in each station field of the harbour and cumulative frequency of consumption can enjoy more preferential incentives.

\section{Conclusion and prospect}

Overall the application of China's IC card electronic certificate for road transportation is still in its early stage, which is mostly concentrated in the industry management. This study summarizes innovative thinking and means in IC card electronic certificate for road transportation in the supervision of passenger station, dangerous goods transport management, governance overload and logistics park and port area management. IC card electronic certificate for road transportation is an important carrier of vehicle and personnel credit information. The credit information management is the key for vehicle and personnel management and service. The future research is more concentrated in the field of industry service is actively exploring using by IC card. For example, by IC card electronic license for road transportation as the carrier, the establishment of national freight service alliance, and oil retail companies, vehicle maintenance and rescue companies, telecommunications companies, insurance companies and other value-added service providers identified for the cardholder to provide the relevant preferential policies and enjoy the group purchase benefit. In addition, it can also be linked to the transport business households and practitioners credit record and enjoy the highways, transport station, valueadded service providers to provide preferential service standards and credit records of good cardholders will enjoy greater discount preferential service. Otherwise it has no right to enjoy, and even be included in the blacklist to accept severe punishment. This will effectively promote the transport industry practitioners to regulate business, honest and trustworthy, truly scientific management and services to achieve the perfect unity.

\section{References}

1. X. Liu. Transport Business China 5, Adhere to Market Leading to Promote the Intensive Development of Road Transport Industry. 18-20 (2014)

2. W. Kou, S. Poon, E. M. Knorr. Payment Technologies for E-Commerce, Smart Cards and Applications. 95-126, (2003)

3. Ministry of Transport of the People' $s$ Republic of China. China Communications Press, JT/T 825-2012 IC card license for road transportation. (2012)

4. X. Zhang, L. Liu, L. Zhang, China ITS Journal 9, Research and application of IC card technology in transportation. 131-134, (2012)

5. L. Wang, X. Yang, N. Shen, L. Li. Highway \& Automotive Applications 2, Research on the application of IC card in Shanxi Province. 79-81, (2013)

6. X. Wei. Modern Industrial Economy and Informationization 46, The Role of IC Road Transportation Certificate in Management of Highway Transportation Industry. 90-100 (2013) 